\section{Case Report}

Volume : 1 , Issue :1

April 2017

Pages: 1 - 4

\title{
Acetaminophen toxicosis in a cat
}

\author{
Burçak Özkan*
}

\begin{abstract}
Acetaminophen causes serious problems as toxication in cats in spite of being an effective and reliable analgesic and antipyretic in humans. A six months-old female cat suffering from cough was presented to examination to International Pet Hospital/Tirana/Albania when no result was obtained after one acetaminophen tablet had been administered in order to heal the disease. Depression, grey and cyanotic mucous membranes and tongue, tachypnea, tachycardia, hypothermia were primary clinical signs. The color of blood and urine were darkened. On the basis of anamnesis and clinical findings, a definitive diagnosis of acetaminophen toxicosis was made. Therapeutic protocol contained blood transfusion, $\mathrm{N}$-Acetylcysteine, ascorbic acid, cimeditine and IV fluid therapy. Patient's blood samples were collected for haemogram and serum analyses with regular intervals was cured with appropriate treatment.
\end{abstract}

Received: 25.02 .2017

Accepted: 03. 04.2017

Available online: 13.04.2017

Keywords: cat, acetaminophen, toxicosis

DOI: $10.30704 /$ http-www-jivs-net.295062

To cite this article: Özkan, B. (2017). Acetaminophen toxicosis in a cat. Journal of Istanbul Veterinary Sciences, 1(1), 1-4.

Abbreviated Title: J Ist Vet Sci

\section{Introduction}

Acetaminophen also known as paracetamol is an effective and reliable analgesic and antipyretic for human use. It is strongly associated with serious problems in companion animals and toxication have been commonly reported. Its use is definitively contraindicated in cats since a dosage applied with success in another species has lethal causes (Sellon, 2013). The most common cause in acetaminophen poisoning is the incorrect applications by the owner without prior consultation with the veterinarian (Denzoin Vulcano et al., 2013). Therefore, acetaminophen intoxication represents a common cause of feline and canine toxicosis reported to veterinary hospitals (Allen, 2003). Acetaminophen in animal species (cats, dogs, ferrets, birds, pigs, primates, and many others) is associated with hepatotoxicity because of the metabolite $\mathrm{N}$-acetyl-p-benzoquinoneimine (McConkey et al., 2009). It is rapidly absorbed from the gastrointestinal tract. Peak plasma concentrations are usually seen within an hour. The metabolism of drug involves two major conjugation pathways in most species. Both involve cytochrome P450 metabolism, followed by glucuronidation or sulfation (Khan, 2016).
$\mathrm{N}$-acetyl-p-benzoquinoneimine binds to glutathione (GSH) in the physiologic conditions or at therapeutic doses. On the other hand, at toxic doses, sulfate and glucuronosyl transferases become saturated and increased $\mathrm{N}$-acetyl-p-benzoquinoneimine production occurs (Davis et al., 1974). As a result of this, N-acetyl-pbenzoquinoneimine binds covalently to cysteine groups on other hepatocellular proteins leading to cell death (Landin et al., 1996). In dogs and cats, overdose acetaminophen primarily causes methemoglobinemia and hemolysis (McConkey et al., 2013). However, there are situations in which veterinarian may prescribe a specific dosage of acetaminophen for dogs. Clinical signs of acute toxicity are usually not seen unless the dosage of acetaminophen exceeds $100 \mathrm{mg} / \mathrm{kg}$ in dogs. On the other hand, cats are more sensitive to acetaminophen toxicosis, because they are deficient in glucuronyl transferase and therefore have limited capacity to glucuronidate this drug (Khan, 2016). Therefore cats are 7 to 10 times more susceptible to acetaminophen toxicity than dogs are. Acetaminophen toxications have been commonly reported in companion animals in worldwide. Therefore we aimed to discuss the healing procedure of our patient with the case report below. 


\section{Case Presentation}

The patient was a 6-months-old female cat weighing 1.3 kg. (Figure 1). The owner reported a cough enduring for a week. Patient's owner explained 1 Parol tablet $\AA$ (Atabay) was swallowed (acetaminophen $/ 500 \mathrm{mg}$ ) and she had become distressed in 1-2 hours.

\section{Clinical examination and findings}

Initial physical examination revealed that the cat was depressed. The oral and nasal mucosal membranes and the tongue were grey and cyanotic. Tachypnea, tachycardia and hypothermia were detected. Heart and respiratory rates were respectively $45 / \mathrm{min}$. (reference value: $16-40 / \mathrm{min}$.), 160/min. (reference value:120$140 /$ min.) and rectal temperature was $36.5{ }^{\circ} \mathrm{C}$ (reference value: $37.5-39.1^{\circ} \mathrm{C}$ ). The color of the blood was dark red-brown and the urine also had a dark color. Haemogram and biochemical analyses were realized on days $3,10,15$ and 30 apart from the one realized at her arrival. The pathologic values and their situation in the following days are represented in Table 1. Approximately 8 hours after the beginning of the treatment, a facial edema especially remarkable around the orbitas and lips developed. But it disappeared progressively within a few hours as the cure went on. An amelioration of mucosal grey discolor was noted with the edema disappearing. On day 3, mucosa membranes turned icteric. Appetite and water intake were diminished. Blood analyses showed that RBC and HGB were decreased while the liver enzymes and bilirubin levels were increased. Blood transfusion was performed. An amelioration of respiratory and cardiac rates were observed following the transfusion as the appetite got better. Mucous membranes of icteric appearance was replaced by a healthy color. Respiratory and cardiac rates normalized. RBC and HGB analyses performed 12 hours after blood transfer were respectively $5.0 \times 106 \mu \mathrm{L}$ and $7.2 \mathrm{~g} / \mathrm{dL}$.

Diagnosis: On the basis of anamnesis and findings, acetaminophen toxicosis was definitively diagnosed.

Treatment: Primarily the cat was hospitalized and the body temperature was normalized with the help of external heat. The primal objective was the removal of the cause, the compensation of the systemic damage accompanied by hepatic dysfunction and to establish hepatic regeneration (Ettinger and Feldman, 2010). No therapeutic emesis was induced since the drug had already been indigested. The medical intervention was so carefully realized that no harm was made since the stress related to the methemoglobinemia could cause the death.

First of all, N-Acetylcysteine (Fluimucil ®; Zambon) at a loading dose of $280 \mathrm{mg} / \mathrm{kg}$ PO diluted to a $5 \%$ solution in water was administered (Anvik, 1984). The second dose was $140 \mathrm{mg} / \mathrm{kg}$ and the follows were 70 $\mathrm{mg} / \mathrm{kg} / \mathrm{q} 6 \mathrm{~h}$. (Ettinger and Feldman, 2010; Plunkett, 2001; Tennant, 2005). IV fluid therapy was planned in order to maintain hydration, electrolyte balance, vascular volume and renal and hepatic perfusion (Sellon, 2013). The fluid therapy began with $0.9 \% \mathrm{NaCl}$, continued with the use of $2.5 \%$ dextrose $+0.45 \% \mathrm{NaCl}$ because of the hepatic enzyme increase (Richardson, 2000). Ascorbic acid (Vitamin-C) (Redoxon®; Roche) was given at $30 \mathrm{mg} / \mathrm{kg} \mathrm{q} 6 \mathrm{~h}$ in order to convert methemoglobin back to hemoglobin (Richardson, 2000; Tennant, 2005). Cimetidine (Cimetidine ${ }^{\circledR}$; Wallgreens) was added into the protocol at a dose of $10 \mathrm{mg} / \mathrm{kg} P O$ q6h. The dose was arranged as $5 \mathrm{mg} / \mathrm{kg}$ IM q12h when liver enzymes increased since careful use is suggested in hepatic dysfunction (Steenbergen, 2003). Blood transfusion was performed on 3th day when anemia was seen (Plunkett, 2001; Sellon, 2013).

\section{Discussion}

In most mammals, acetaminophen is eliminated by two pathways called "glucoronidation" and "sulfonidation". Both of these processes produce inactive metabolites eliminated by the kidneys (Richardson, 2000). In case these pathways become saturated, there is an alternative one breaking down the acetaminophen to a toxic metabolite (Allen, 2003) called "N-Acetylparabenzequinoneimine (NAPQUI)". NAPQUI's toxic influences are restricted by glutathione distributed in mammalian tissues. When cellular glutathione become less than $70 \%$ of normal, NAPQUI binds to cellular proteins and membranes and leads to hepatocyte injury (Allen, 2003). Cats are extremely sensitives to acetaminophen toxication due to the lack of glucoronyl transferase and glutathion stores, they form glucoronides with a lot of compounds slowly or not at all (Allen, 2003; Ettinger and Feldman, 2010). The absence of this biochemical step causes more drug to be conjugated to sulfates since the sulfation pathway has a 
finite capacity, lower than the other species. This is why acetaminophen is allowed to persist in the blood and to be metabolized to NAPQUI once the sulfation pathway reaches its capacity. High levels of acetaminophen suppress glutathione synthesis at the same time NAPQUI depletes gluathione stores (Steenbergen, 2003). NAPQUI causes hepatocyte necrosis (Steenbergen, 2003). Erythrocytes are predisposed to NAPQUI effects in cats (Allen, 2003) which causes oxidative stress and ferrous iron $(\mathrm{Fe} 2+)$ to be oxidized to ferric iron $(\mathrm{Fe} 3+)$ converting hemoglobin to methemoglobin (Richardson, 2000). As the cats possess methemoglobin reductase deficiency in their erythrocytes, methemoglobinemia occurs earlier. Hemoglobin oxidation leads to "Heinz Bodies" production causing erythrocyte fragility and hemolytic anemia. Tissue anoxia is due to methemoglobin suffering from oxygen transporting deficiency. It is the methemoglobinemia and hemolytic anemia that account for the respiratory distress, depression, dark brown or pale mucous membranes, icterus and pigmenturia. Feline hemoglobin instability causes Heinz bodies to be formed easily with the help of sulfhydryl groups (Allen, 2003). When they are overwhelmed by increased methemoglobin production, erythrocyte glutathion becomes an antioxidant reserve to assist the methemoglobin reductase enzymes. This glutathion depletion alters the protective antioxidant effect and leads to exaggerated methemoglobin formation. Feline erythrocytes are prone to oxidative changes and cats have depleted erythrocyte glutathion levels in acetaminophen toxication (Anvik,1984). In spite of some researchers reporting that cats could tolerate a low dose (Ettinger and Feldman, 2010), toxic dose is explained as $50-60 \mathrm{mg} / \mathrm{kg}$ and cats die from acetaminophen intake even a very small dose is ingested (Plunkett, 2001; Sellon, 2013). Despite having swallowed a quite large amount, the cat described here survived probably because of the early treatment that prevented hepatic damage. The clinicopathological symptoms developed in our patient were similar to those described elsewhere (Ettinger and Feldman, 2010).

The excessive accumulation of methemoglobin causes hemoglobin denaturation, Heinz body formation, increased RBC osmotic fragility and hemolytic anemia followed by icterus, hemoglobinemia, tissue anoxia and cyanosis (Anvik, 1984). Methemoglobinemia is a reversible injury to hemoglobin and leads to decreased oxygen carrying capacity and characteristic brown discoloration of blood and mucous membranes. The lethal effect is cyanosis resulting from severe methemoglobinemia occurring 24 to 36 hours before hepatic damage (Ettinger and Feldman, 2010). Tachypnea and cyanosis point out methemoglobinemia while the edema developed after a few hours the ingestion of the drug seems to be concordant with the literature. The pathogenesis of the edema was related to methemoglobin derived anoxia leading to augmented capillary wall permeability (Anvik, 1984). Laboratory findings of hepatotoxicity generally develops 24-36 hours post-ingestion. Thus, hepatic enzymes should be monitored carefully (Richardson, 2000). Significant abnormalities of serum biochemistry included increased ALT, AST, total bilirubin and direct bilirubin. The elevated hepatic enzymes were probably the result of hepatic damage. The bilirubin increase was thought to be a result of accelerated RBC destruction. The slight decrease accompanying the therapy procedure suggested that there was no ongoing hepatic damage. Neither re-examination on 30th day nor the telephone follow-up next month reported any continuing problem. Serum chemistry abnormalities were concordant with the data. Progressive increase in hepatic enzymes is defined as characteristic, which supported the clinical biochemical representation of our patient (Ettinger and Feldman, 2010).

Toxicity studies suggest that acetaminophen ingestion as high as $140 \mathrm{mg} / \mathrm{kg}$ can be treated successfully if therapy is initiated within four to six hours of ingestion (Anvik,1984). We think that the early medical intervention provided in our case was the most important factor that helped our patient to survive. Neither emesis nor gastric lavage was performed since the toxication related to drug ingestion had began more than two hours before her arrival. The physical activity of the patient was restricted as suggested in order to prevent stress (Steenbergen, 2003). N-acetylcysteine containing a high number of sulfhydral groups counters acetaminophen toxicity by augmenting serum sulfate and supplies of glutathione. The reason why the oral route was preferred was that acetylcysteine is absorbed effectively via gastrointestinal mucosa when administered orally and reaches the liver via portal circulation for detoxification (Ettinger and Feldman, 2010). It is suggested to organize the therapy schedule according to the amount indigested and clinical findings (Richardson, 2000). We administered 12 total dose in our case. Blood transfusion improves oxygen-carrying capacity of blood in severe cases (Plunkett, 2001; Sellon, 2013). The decrease occurred in our patient's hemogram values and anemia normalized when blood transfusion was applied. 
Özkan B. 2017/ Journal of Istanbul Veterinary Sciences. Volume 1, Issue 1, pp: 1-4.

Table 1. Hematologic and biochemical values represented with abnormalities during the treatment

\begin{tabular}{lcccccc}
\hline \multirow{2}{*}{ Blood Parameters } & \multicolumn{3}{c}{ Treatment Days } & \multirow{2}{*}{ Reference Values } \\
\cline { 2 - 5 } & 1 & 3 & 10 & 15 & 30 & $6-10$ \\
RBC $\left(10^{6} / \mu \mathrm{L}\right)$ & 7.5 & 1.7 & 6.2 & 6.4 & 7.8 & $9.5-15$ \\
HGB (g/dL) & 12 & 2.3 & 9.1 & 10.8 & 10.9 & $0.0-0.4$ \\
Total Bilirubin (mg/dL) & 0.2 & 4.2 & 0.7 & 0.3 & 0.3 & $0.0-0.1$ \\
Direct Bilirubin (mg/dL) & 0.1 & 3.8 & 0.4 & 0.1 & 0.1 & $28-76$ \\
ALT (IU/L) & 55 & 290 & 82 & 80 & 50 & $5-55$ \\
AST (IU/L) & 45 & 132 & 78 & 73 & 46 &
\end{tabular}

RBC: Red Blood Cell, HGB: Hemoglobin, ALT: Alanine aminotransferase, AST: Aspartate aminotransferase

Cimetidine reduces acetaminophen metabolism by inhibiting cytochrome P-450 oxidation in the liver (Richardson, 2000). It is also used to reduce toxic metabolite production. In spite of its controversial use due to its debatable hepatotoxic influence, we added cimetidine in our protocol since NAC and ascorbic acid combination's therapeutic use was proven to be more effective than the use of these agents alone (Plunkett, 2001; Richardson, 2000). Researches show a higher incidence of acetaminophen induced methaemoglobinemia in cats fed a diet rich in propylene glycol. Wet can food contains 7-13\% propyleneglycol which may induce methemoglobinemia or Heinz body formation. The pre -existence of RBC damage caused by propylene glycol consumption may contribute to increased erythrocyte sensitivity to oxidative stress related to acetaminophen toxication (Steenbergen, 2003).

Highly containing sulfhydryl group substrate, kitten food was preferred at the beginning. When hepatic enzymes increased, because of this augmentation and

\section{References}

Allen, A. L. (2003). The diagnosis of acetaminophe toxicosis in a cat. Canadian Veterinary Journal, 44, 509-510.

Anvik, J. O. (1984). Acetaminophen toxicosis in a cat. Canadian Veterinary Journal, 25, 445-447.

Denzoin Vulcano, L. A., Confalonieri, O., Franci, R., Tapia, M. O., \& Soraci, A .L. (2013). Efficacy of free glutathione and niosomal glutathione in the treatment of acetaminopheninduced hepatotoxicity in cats. Open Veterinary Journal, 3(1). 56-63.

Ettinger, S. J. \& Feldman, E. C. (2010). Textbook of veterinary internal medicine vol 2. Philadelphia, US: W.B. Saunders.

Khan, S. A. (2016). Analgesics (Toxicity). In S. E. Aiello, \& M. A. Moses (Eds), The Merck Veterinary Manual 11th ed.

Retrieved from http://www.merckvetmanual.com/toxicology/ toxicities-from-human-drugs/analgesics-toxicity.

Landin, J. S., Cohen, S. D. \& Khairallah, E. A. (1996). Identification of a54-kDa mitochondrial acetaminophenbinding protein as aldehydedehydrogenase. Toxicology and with the aim of prevent renal injury the cat was fed K/ D (Plunkett, 2001).

As a result, we concluded that it could be possible to obtain a satisfactory response with the help of early treatment, appropriate antidote and supportive therapy.

Before treatment (icteric view) After treatment

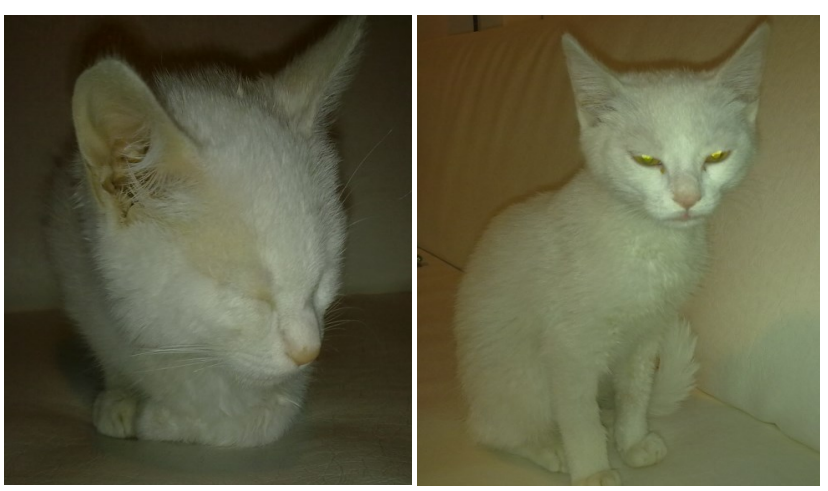

Figure 1. The appearance of the cat.

Applied Pharmacology, 141(1), 299-307.

McConkey, S. E., Grant, D. M., \& Cribb, A. E. (2009). The role of para-aminophenol in acetaminophen-induced methemoglobinemia in dogs and cats. Journal of Veterinary Pharmacology and Therapeutics, 32(6), 585-95.

Plunkett, S. J. (2001). Emergency procedures for the small animal veterinarian 2 nd ed. London, W.B. Saunders.

Richardson, J. A. (2000). Management of acetaminophen and ibufrofen toxicoses in dogs and cats. Journal of Veterinary Emergency and Critical Care, 10(4), 285-291.

Sellon, R. K. (2013). Acetaminophen, In M. E. Peterson \& A. P. Talcott (Ed). Small animal toxicology 3rd ed. (pp. 423-429). St Louis, US: W.B. Saunders.

Steenbergen, V. (2003). Acetaminophen and Cats. Retrieved from: http://aspcapro.org/sites/pro/files/avettech_0103_0.pdf

Tennant, B. (2005). BSWA Small Animal Formulary 5th ed. Wetherby (Leeds), UK: Veterinary Publishing Company. 